\title{
IMBEDDING THE STABLE HOMOTOPY CATEGORY
}

\author{
BY DONALD W. KAHN ${ }^{1}$
}

\author{
Communicated by William Browder, May 1, 1967
}

The stable homotopy categories have attracted the attention of topologists for more than a decade. The most basic such category, ews sh, is the category of finite CW-complexes and stable (under suspension) homotopy classes of maps. As a category, it has many unusual properties: it is non-Abelian, although sums agree with products; every object is projective, etc. There is a sizeable literature (see $[1],[2],[3],[5]$, and [6]).

However, a general structure theorem for this category is lacking. A full algebraic description of the category seems out of reach, because it would describe the stable homotopy of spheres, for example. But we may ask for a description modulo some basic geometry. It appears to the author that, for the present, an ideal description would consist of some algebraic structure defined in terms of the stable homotopy ring of spheres, $G_{*}$ (see [7]).

We announce here an imbedding of the category ews in a quotient of the category of exact couples of $G_{*}$-modules. Details of these results, and the generalizations of $\$ 3$, will appear in future publications.

1. Let $\mathcal{E C}_{R}$ be the category of bigraded exact couples (4) of modules, over the graded ring $R\left(1 \in R^{0}\right)$. $R$ acts on the second summand of such a module via maps

$$
R^{s} \times A^{p, q} \rightarrow A^{p, q+s} .
$$

We assume $A^{p, q}=0$, if $p<0 ; B^{p, q}=0$, if $q<0$. A morphism in this category is a couple map, which is also a collection of $R$-linear maps. For such couples, $\langle A, B, f, g, h\rangle$, we assume $f, g$, and $h$ have bidegrees $(+1,-1),(0,0),(-1,0)$.

There is a homotopy theory in $\mathcal{E C}_{R}$ given by

Definition 1. If $\langle A, B, f, g, h\rangle \in \mathcal{E} \mathfrak{C}_{R}$, set

$$
\begin{array}{rlrl}
\bar{A}^{p, q} & =A^{p, q} \oplus B^{p, q} & \bar{f}(x, y) & =(f(x), 0), \\
\bar{B}^{p, q} & =B^{p, q} \oplus B^{p, q} \oplus B^{p-1, q}, & \bar{g}(x, y) & =(g(x)-y, y,-g h(y)), \\
\bar{h}(x, y, z) & =(h(x+y), z+g h(y)) .
\end{array}
$$

\footnotetext{
${ }^{1}$ Work partially supported by contract (NSF) GP-5252.
} 
It is easy to see that $\langle\bar{A}, \bar{B}, \bar{f}, \bar{g}, \bar{h}\rangle \in \mathcal{E} \mathcal{C}_{R}$, and that when we define couple maps

$$
\left\langle r^{i}, s^{i}\right\rangle:\langle A, B, f, g, h\rangle \rightarrow\langle\bar{A}, \bar{B}, \bar{f}, \bar{g}, \bar{h}\rangle, \quad i=1,2,
$$

$$
\text { by } \begin{aligned}
r^{1}(x) & =(x, 0), \quad s^{1}(x)=(x, 0,0), \\
r^{2}(x) & =(x, g(x)), \quad s^{2}(x)=(0, x, 0),
\end{aligned}
$$

we see that $\langle\bar{A}, \bar{B}, \bar{f}, \bar{g}, \bar{h}\rangle$ plays the role of Cartesian product with the unit interval, and $\left\langle r^{i}, s^{i}\right\rangle$ are canonical injections.

Definition 2. Given two couple maps $\left\langle u^{i}, v^{i}\right\rangle:\langle A, B, f, g, h\rangle$ $\rightarrow\langle C, D, l, m, n\rangle$, write $\left\langle u^{1}, v^{1}\right\rangle \sim\left\langle u^{2}, v^{2}\right\rangle$ (that is to say they are "homotopic"), if there exists a map

$$
\langle U, V\rangle:\langle\bar{A}, \bar{B}, \bar{f}, \bar{g}, \bar{h}\rangle \rightarrow\langle C, D, l, m, n\rangle
$$

so that

$$
\left\langle u^{i}, v^{i}\right\rangle=\langle U, V\rangle \cdot\left\langle r^{i}, s^{i}\right\rangle, \quad i=1,2 .
$$

This is an equivalence relation, and we define the quotient category to be $\varepsilon \mathfrak{C}_{R} h$.

2. Let $\mathrm{e} w$ be the category of finite (based) $\mathrm{CW}$-complexes and (based) $\mathrm{CW}$-maps. ewsh is the quotient of this category, taking stable homotopy classes of maps.

Definition 3. Define a functor $\mathfrak{F}: \mathcal{C} \mathscr{W} \rightarrow \mathcal{E} \mathfrak{C}_{G *}\left(\right.$ recall $\left.G_{*}=\pi_{*}^{s}\left(S^{0}\right)\right)$ by $\mathscr{F}(X)=\langle A, B, f, g, h\rangle$ with

$$
A^{p, q}=\pi_{p+q}^{s}\left(X^{p}\right), B^{p, q}=\pi_{p+q}^{s}\left(X^{p} / X^{p-1}\right) ;
$$

$X^{p}$ means $p$-skeleton, $s$ refers to stable, and the maps are the obvious ones.

THEOREM 1. a. F is a functor from $\mathfrak{C}$ W to $\mathfrak{E C}_{G *}$.

b. $B^{p, *}$ is a free bigraded $G_{*}$-module, whose dimension is the number of p-cells of $X$.

c. $B_{n}^{*, *} \Rightarrow \pi_{*}^{s}(X)$.

d. $\mathcal{F}(X)=\mathcal{F}(Y)$ implies there is $n \geqq 0$ and a CW-homotopy equivalence $f: \Sigma^{n} X \rightarrow \Sigma^{n} Y$

e. If $X \in \mathcal{e} W, \mathcal{F}(X)=\langle A, B, f, g, h\rangle$, then

$$
\mathfrak{F}(X \times I)=\langle\bar{A}, \bar{B}, \bar{f}, \bar{g}, \bar{h}\rangle .
$$

The canonical injections are induced by inclusion of $X$ as faces of $X \times I$.

It follows now that $\mathscr{F}$ induces a functor

$$
\mathcal{F}_{0}: \mathfrak{e} w_{s h} \rightarrow \mathcal{E C}_{G_{*}} h .
$$


THEOREM 2. $\mathcal{F}_{0}$ is an imbedding, in that

a. If $X$ and $Y$ do not have the same stable homotopy-type, $F_{0}(X)$ $\neq \mathfrak{F}_{0}(Y)$.

b. If $f, g: X \rightarrow Y$ are not stably-homotopic, $\mathfrak{F}_{0}(\{f\}) \neq \mathcal{F}_{0}(\{g\})$ Modulo maps on which $\mathcal{F}$ is zero.

3. If the imbedding $F_{0}$ is to have significance, the bigger category $\mathcal{E}_{G_{*}} h$ should itself have interesting properties. In fact, one may show that it is possible to do much of algebraic topology in $\mathcal{E} \mathfrak{C}_{R} h$, for any $R$. Homology, cohomology, and homotopy modules are defined, and we prove general versions of the theorems of Hurewicz, Whitehead, etc.

The basic open problems are which maps in $\mathcal{E C}_{G_{*}} h$ come from $\mathfrak{F}_{0}$, and whether $\mathcal{E} \mathcal{C}_{R} h$ has cone constructions.

\section{BIBLIOGRAPHY}

1. J. F. Adams, Lectures on stable homotopy theory, Springer-Verlag, Berlin, 1964.

2. M. Boardman, Stable homotopy theory, University of Warwick, 1966.

3. P. Freyd, Stable homotopy. LaJolla Conference on Category Theory, Springe rVerlag, Berlin, 1965.

4. W. S. Massey, Exact couples in algebraic topology, Ann. of Math. 56 (1952), 363396 and 57 (1953), 248-286.

5. D. Puppe, (a) On the formal structure of stable homotopy theory, Colloquium at Aarhus, Proceedings, 1962.

(b) Stabile homotopietheorie, Math Ann. 169 (1967), 243-274.

6. E. Spanier and J. H. C. Whitehead, A first approximation to homotopy theory, Proc. Nat. Acad. U.S.A. 39 (1953), 655-660.

7. H. Toda, Composition methods in homotopy groups of spheres, Princeton Univ. Press, Princeton, N. J., 1962.

University of Minnesota 